\title{
SARS-CoV-2 infection in children with rheumatic disease: Experience of a tertiary referral center
}

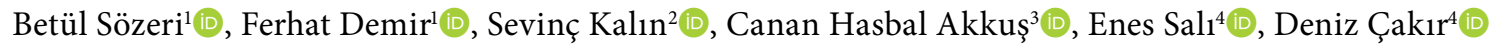 \\ ${ }^{1}$ Department of Child Health and Diseases, Pediatric Rheumatology, Ümraniye Training and Research Hospital, Istanbul, Turkey \\ ${ }^{2}$ Department of Radiology, Pediatric Radiology, Ümraniye Training and Research Hospital, Istanbul, Turkey \\ ${ }^{3}$ Department of Child Health and Diseases, Department of Pediatrics, Ümraniye Training and Research Hospital, Istanbul, Turkey \\ ${ }^{4}$ Department of Child Health and Diseases, Pediatric Infectious Disease, Ümraniye Training and Research Hospital, Istanbul, Turkey
}

\section{ABSTRACT}

Objectives: In this study, we present our clinical severe acute respiratory syndrome-coronavirus 2 (SARS-CoV-2) experience in patients with childhood rheumatic disease during novel coronavirus-2019 (COVID-19) pandemic.

Patients and methods: A total of 87 patients (50 males, 37 females; median age: 12 years; range, 6.6 to 16 years) suspected of having COVID-19 at our pediatric rheumatology clinic between March $11^{\text {th }}$ and October $15^{\text {th }} 2020$ were retrospectively analyzed. Demographic and clinical features, treatments, laboratory results, imaging findings, and clinical outcomes of the patients diagnosed with COVID-19 and/or multisystem inflammatory syndrome in children (MIS-C) were retrieved from the medical records. The diagnosis of SARS-CoV-2 infection was made based on the reverse transcriptase-polymerase chain reaction test.

Results: The most common rheumatic diseases were juvenile idiopathic arthritis and familial Mediterranean fever (35.6\% and $34.5 \%$, respectively). Twenty-six of these patients were treated with biological disease-modifying anti-rheumatic drugs. SARS-CoV-2 infection was tested as positive in 84 (96.5\%) patients. Also, 51 (58.6\%) patients had an epidemiological contact to a person with COVID-19. Eighteen patients met the clinical criteria and diagnosed with MIS-C. The COVID-19 outbreak also caused exacerbation of systemic disease in 56 children due to medication cessation, postponed drug switch, or recurrent viral infection.

Conclusion: Children with rheumatic disease do not appear to present a higher risk of severe COVID-19. The immunosuppressive treatments can be adjusted in case of infection; otherwise, it is not recommended to interrupt the treatments. Physicians should be cautious about the hyperinflammatory syndrome associated with COVID-19 in rheumatic children, which may be severe in this group of patients and may be confused with primary diseases.

Keywords: Biological treatment, COVID-19, SARS-CoV-2, multisystem inflammatory syndrome, pediatric rheumatology.

A large outbreak of a novel coronavirus infection occurred in China in December 2019. ${ }^{1}$ Since the first case was reported on March $11^{\text {th }}$, 2020, about 340,450 confirmed cases have been declared in Turkey. ${ }^{2}$ A total of $2 \%$ of the reported cases is under age $20^{3}$ and the pediatric population seems to be affected lower than adults from this infection.
Although various results have been reported on the subject, it is still unknown whether patients with rheumatic disease, many of whom are on immunosuppression, are at a higher risk of severe novel coronavirus-2019 (COVID-19). ${ }^{3,4}$ In adult studies, the patients with inflammatory arthritis receiving immune suppressive therapies do not show an increased risk of associated

Received: November 28, 2020 Accepted: December 13, 2020 Published online: February 09, 2021

Correspondence: Betül Sözeri, MD. Ümraniye Eğitim ve Araştırma Hastanesi, Çocuk Sağlığı ve Hastalıkları Kliniği, Çocuk Romatolojisi Bölümü, 34764 Ümraniye, İstanbul, Türkiye. Tel: +90 462 - 3775435 e-mail: drbetulsozeri@gmail.com

\section{Citation:}

Sözeri B, Demir F, Kalın S, Hasbal Akkuş C, Salı E, Çakır D. SARS-CoV-2 infection in children with rheumatic disease: Experience of a tertiary referral center. Arch Rheumatol 2021;36(3):381-388. 
complications of severe acute respiratory syndrome-coronavirus 2 (SARS-CoV-2), compared to the general population. ${ }^{5,6}$ However, there is a limited number of data regarding the infection ratio and disease course of SARS-CoV-2 in patients with childhood rheumatic diseases, particularly pediatric cases. In general, children experience a mild form of COVID-19 and have a much lower risk of severe forms than other age groups, according to some reports from multiple countries. $^{7-11}$

Throughout the pandemic, some cases were reported from various countries describing pediatric patients with unusual febrile disease, acute abdominal conditions, encephalopathy, elevated inflammatory markers, and multisystem involvement. ${ }^{12-15}$ The emerging disorderwastermed "pediatric inflammatory multisystem syndrome temporally associated with SARS-CoV-2," or PIMS-TS. ${ }^{14,15}$ This new syndrome was termed as multisystem inflammatory syndrome in children (MIS-C) from The United States Centers for Disease Control and Prevention (CDC) and the World Health Organization (WHO). ${ }^{16,17}$ In recent studies, patients who met this definition were reported. ${ }^{12,13}$

Currently, there are insufficient data on the effects of both the COVID-19 infection and the pandemic period on pediatric rheumatic patients. To address this gap, in this study, we present our experience in children with rheumatic disease during the COVID-19 outbreak.

\section{PATIENTS AND METHODS}

This single-center, retrospective study was conducted at Department of Pediatric Rheumatology, Umraniye Training and Research Hospital, which is a referral center for pediatric rheumatic disease between March $11^{\text {th }}, 2020$ and October $15^{\text {th }}, 2020$. A total of 4,470 patients $(3,122$ in the outpatient clinic, 38 by consultation, and 1,310 via telemedicine) at our clinic during the pandemic were screened. We evaluated 1,310 patients by telemedicine (as reported by parents via phone consultation and was applied a questionnaire form including both for primary disease and suspected symptoms of COVID-19 with global patient/parent activity scores). If there was any suspicion about the disease, the patient was invited to the hospital. A child who met at least one of the following criteria was considered to be a COVID-19-positive patient: (i) The patients who were admitted to the hospital with symptoms of fever and acute respiratory infection (cough and/or respiratory distress) that were not explained for any other reason, and who needed to be hospitalized; (ii) The patients who had at least one of the symptoms of fever, cough or respiratory distress, as well as a history of contact with a patient with COVID-19 infection. ${ }^{18}$ The criteria used for the diagnosis of MIS-C as follows (one had to meet $1+2+3$ ): (i) A children presenting with fever $\left(>38.0^{\circ} \mathrm{C}\right.$ for $\geq 24 \mathrm{~h}$ ), laboratory evidence of inflammation, and evidence of clinically severe illness requiring hospitalization with multisystem $(>2)$ organ involvement (cardiac, renal, respiratory, hematologic, gastrointestinal, dermatologic or neurological); (ii) No alternative possible diagnoses or no obvious microbial cause of inflammation; and (iii) Positive for current or recent SARS-CoV-2 infection by reverse transcriptase-polymerase chain reaction (RT-PCR), serology, or antigen test; or exposure to a suspected or confirmed COVID-19 case within the four weeks prior to the onset of symptoms. ${ }^{16,17}$ The presence of at least one of the RT-PCR or antibody test positivity were required as the inclusion criteria. A contact history to a patient with COVID-19 infection was also required for inclusion, in the absence of positive laboratory or imagining result for COVID-19, in patients with a pre-diagnosis of MIS-C. Finally, a total of 87 patients (50 males, 37 females; median age: 12 years; range, 6.6 to 16 years) suspected of having COVID-19 at our pediatric rheumatology clinic were included (Figure 1). A written informed consent was obtained from each parent and/or legal guardian of the patient. The study protocol was approved by the Umraniye Training and Research Hospital Ethics Committee (approval no/date: B10.1TKH.4.34.H.G.P.0.01/101, 25.05.2020). The study was conducted in accordance with the principles of the Declaration of Helsinki.

Demographic features, medical histories, clinical symptoms, laboratory results, thoracic computed tomography (CT) findings, and clinical 

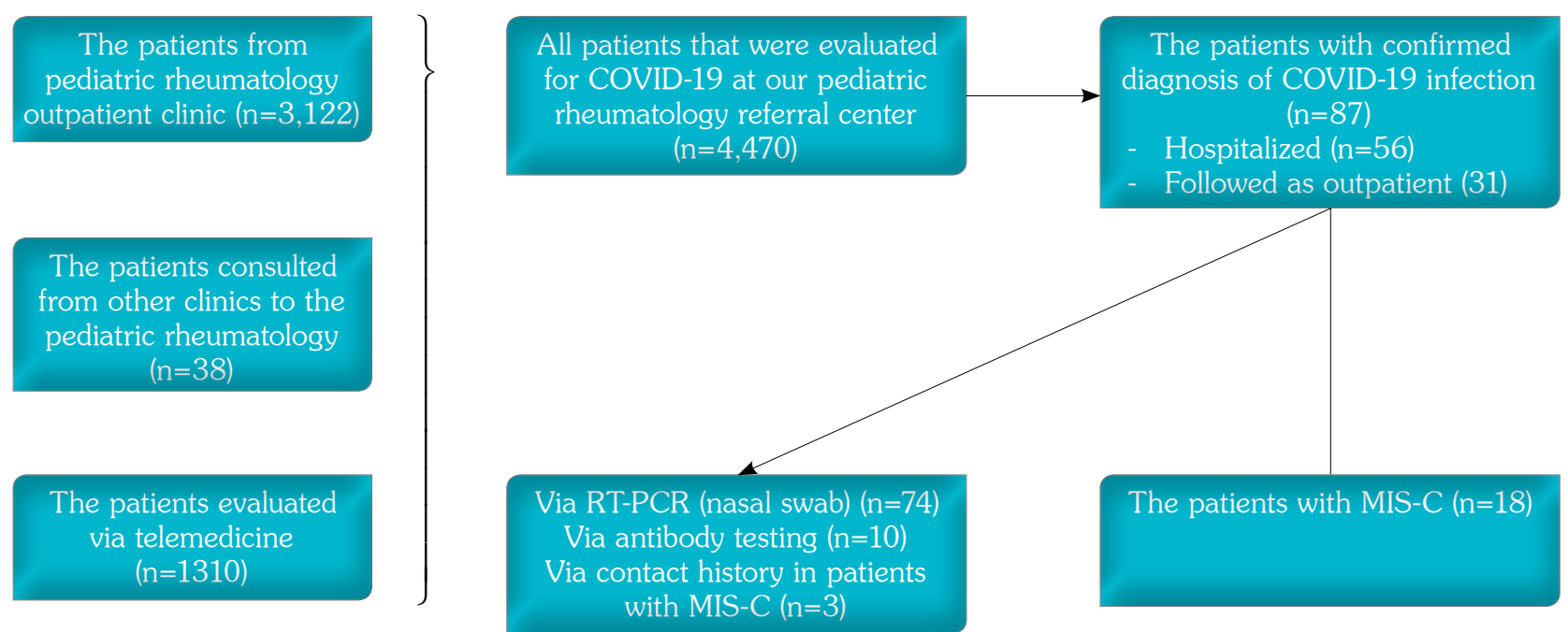

Figure 1. Study flow chart.

COVID-19: Coronavirus-2019; RT-PCR: Reverse transcriptase-polymerase chain reaction; MIS-C: Multisystem inflammatory syndrome in children.

outcomes of patients were retrieved from the medical records. Respiratory samples from the all patients were analyzed with next-generation sequencing or real-time RT-PCR methods for SARS-CoV-2. Thoracic CT examination was done using the Optima ${ }^{\mathrm{TM}}$ CT 660 SE as 128 unit (GE Healthcare, Milwaukee, WI, USA) device, both lung parenchyma $(-700$ to $+1500 \mathrm{HU})$ and mediastinal area (+50 to $+350 \mathrm{HU}$ ) were scanned. Thoracic CT was performed in a supine position without using a contrast agent. In patients who could cooperate, the shooting was done at the end of inspiration.

\section{Statistical analysis}

Statistical analysis was performed using the IBM SPSS version 23.0 software (IBM Corp., Armonk, NY, USA). Continuous variables were expressed in median and interquartile range (IQR), while categorical variables were expressed in number and percentage. The normality of the distribution of continuous variables was assessed using the Shapiro-Wilk test. In univariable analyses, differences in demographic and rheumatic disease-specific features were compared using the chi-square test for categorical variables and Mann-Whitney $\mathrm{U}$ tests for continuous variables. A $p$ value of $<0.05$ was considered statistically significant.

\section{RESULTS}

In this study period, among 4,470 patients, 87 (1.94\%) COVID-19 suspected patients were evaluated. Fifty-six (64.4\%) patients were hospitalized and 31 were followed without hospitalization. Twenty-four (27.6\%) of these patients applied to the clinic for the first time, whereas 63 (72.4\%) were previously diagnosed.

The patients had different rheumatic diseases; mostly juvenile idiopathic arthritis (JIA, 35.6\%) and familial Mediterranean fever (FMF)/other systemic autoinflammatory diseases (SAIDs) (35.6\% and $34.5 \%$, respectively) (Table 1). Fifty (58.6\%) patients had an epidemiologic contact to a person with COVID-19. The primary disease status of these patients were as follows: 78 (89.6\%) were in remission, while nine (10.3\%) had active disease at the time of COVID-19 diagnosis. Twenty-six (29.9\%) patients were treated with biological disease-modifying anti-rheumatic drugs (bDMARDs) and they consisted of $7.2 \%$ of all our patients under biological treatment $(n=360)$. Fifty-six of $(64.3 \%)$ patients had a fever and 20 (23\%) had a fever for five or more days. Gastrointestinal system involvement was in 11 (12.6\%), the respiratory system was in 40 (46\%) and fatigue was in 57 (65.5\%) patients. Cutaneous involvement was seen in five patients including maculopapular rash in two, vasculitic rash in two, and chilblain in one patient (Table 1). 
A total of $63.2 \%$ of the patients had increased C-reactive protein (CRP), 40.2\% had lymphocytopenia $\left(<1,500 / \mathrm{mm}^{3}\right)$, and $26.4 \%$ had elevated D-dimer levels (Table 1). The SARS-CoV-2 infection was confirmed in 84 (96.5\%) patients. The diagnosis was confirmed by RT-PCR in 74 patients and by antibody test in 10. The remaining three patients consisted of those who met the MIS- $\mathrm{C}$ diagnostic criteria and had a contact history with a COVID-19-infected person. Thoracic CT was performed in a total of
45 patients and 28 (62.2\%) of these patients had at least one radiological sign (bilateral, peripheral and/or multilobar involvement, lower segment and/or posterior distribution, mosaic pattern, ground glass opacification, etc.) supportive for COVID-19.

Eighteen (20.7\%) patients met the MIS-C clinical criteria. Nine of them had also hypotension and seven patients were admitted the intensive care unit due to shock and severe end-organ

Table 1. Characteristics of patients with preliminary diagnosis of COVID-19 according to the biological treatment group

\begin{tabular}{|c|c|c|c|c|c|c|c|c|c|}
\hline & \multicolumn{3}{|c|}{ All patients $(n=87)$} & \multicolumn{3}{|c|}{$\begin{array}{l}\text { The patients under } \\
\text { biological treatment }(n=26)\end{array}$} & \multicolumn{3}{|c|}{$\begin{array}{l}\text { The patients without } \\
\text { biological treatment }(n=61)\end{array}$} \\
\hline & $\mathrm{n}$ & $\%$ & Median & $\mathrm{n}$ & $\%$ & Median & $\mathrm{n}$ & $\%$ & Median \\
\hline Age (year) & & & 12 & & & 12 & & & 11.3 \\
\hline \multicolumn{10}{|l|}{ Sex } \\
\hline Female & 37 & & & 12 & & & 25 & & \\
\hline Male & 50 & & & 14 & & & 36 & & \\
\hline \multicolumn{10}{|l|}{ Clinical manifestations } \\
\hline Fatigue & 57 & & & 18 & & & 39 & & \\
\hline Fever $\left(>38.2^{\circ} \mathrm{C}\right)$ & 56 & & & 17 & & & 39 & & \\
\hline Cough and/or respiratory distress & 40 & & & 15 & & & 25 & & \\
\hline Gastrointestinal manifestations & 11 & & & 2 & & & 9 & & \\
\hline Mucocutaneous involvement & 5 & & & 1 & & & 4 & & \\
\hline Presence of MIS-C & 18 & & & 5 & & & 13 & & \\
\hline Hospitalization & 56 & 64.4 & & 20 & 76.9 & & 36 & 59 & \\
\hline \multicolumn{10}{|l|}{ Admission status } \\
\hline Old registered patient & 63 & & & 26 & & & 37 & & \\
\hline First admission/consultation to PR & 24 & & & - & & & 24 & & \\
\hline \multicolumn{10}{|l|}{ Primary systemic disease of patients } \\
\hline FMF/other* SAID & 30 & & & 8 & & & 22 & & \\
\hline JIA & 31 & & & 16 & & & 15 & & \\
\hline SLE & 2 & & & - & & & 1 & & \\
\hline Vasculitis & 7 & & & 2 & & & 5 & & \\
\hline Other & 17 & & & 0 & & & 17 & & \\
\hline \multicolumn{10}{|l|}{ Concomitant systemic treatment } \\
\hline Colchicine & 29 & & & 7 & & & 22 & & \\
\hline Methotrexate & 25 & & & 10 & & & 15 & & \\
\hline Etanercept & 4 & & & 4 & & & - & & \\
\hline Adalimumab & 5 & & & 5 & & & - & & \\
\hline Infliximab & 2 & & & 2 & & & - & & \\
\hline Anti-IL 1 & 12 & & & 12 & & & - & & \\
\hline Anti-IL 6 & 3 & & & 3 & & & - & & \\
\hline \multicolumn{10}{|l|}{ Laboratory\# } \\
\hline Increased CRP (>0.5 mg/dL) & 55 & 63.2 & & 14 & 53.8 & & 41 & 67.2 & \\
\hline Lymphopenia $\left(<1,500 / \mathrm{mm}^{3}\right)$ & 35 & 40.2 & & 12 & 46.2 & & 23 & 37.7 & \\
\hline Increased D-Dimer (>500 ng/mL) & 23 & 26.4 & & 6 & 23.1 & & 17 & 27.8 & \\
\hline Ferritin level (ng/mL) & & & 200 & & & 70 & & & 532 \\
\hline Hyperferritinemia (>684 ng/mL) & 15 & 17.2 & & 4 & 15.4 & & 11 & 18 & \\
\hline Increased IL-6 $(>5.9 \mathrm{pg} / \mathrm{mL})^{* * *}$ & 18 & 72 & & 8 & 32 & & 10 & 40 & \\
\hline
\end{tabular}

MIS-C: Multisystem inflammatory syndrome in children; PR: Pediatric rheumatology; FMF: Familial Mediterranean fever; SAID: Systemic autoinflammatory diseases; JIA: Juvenile idiopathic arthritis; SLE: Systemic lupus erythematosus; CRP: C-reactive protein; IL: Interleukin; \# It indicates the proportion among the total number of individuals examined with a specified laboratory parameter; * Other SAIDs: Mevalonate kinase deficiency or cryopyrin-associated periodic syndrome; ${ }^{* * *}$ IL-6 levels were evaluated in 25 patients. 
illness. The general characteristics of them were as follows: 10 of them were males and the median age was 11.4 years who were younger than the others. A total of $44.4 \%(n=8)$ of the MIS-C patients presented with Kawasaki disease or atypical Kawasaki disease. Five patients were diagnosed and treated with biological drugs before admission. Previous biological treatments of these patients were ceased. There was no significant difference in the treatment strategies of MIS-C patients who previously received or did not receive biological treatment. All cases treated with intravenous immunoglobulin (IVIG), 14 patients received glucocorticoids, two received tocilizumab an interleukin (IL)-6 inhibitor, and four received an IL-1Ra inhibitor (anakinra). Three patients (16.6\%) had cardiovascular involvement and they received vasoactive support. Four patients were also treated with plasmapheresis. Three (16.6\%) patients received invasive mechanical ventilation and a patient received extracorporeal membrane oxygenation support (ECMO) (Table 2). The ECMO

Table 2. Characteristics of patients with and without the multisystem inflammatory syndrome in children

\begin{tabular}{|c|c|c|c|c|c|c|c|}
\hline & \multicolumn{3}{|c|}{ The patients with MIS-C $(\mathrm{n}=18)$} & \multicolumn{3}{|c|}{ Other patients $(n=69)$} & \multirow[b]{2}{*}{$p$} \\
\hline & $\mathrm{n}$ & $\%$ & Median & $\mathrm{n}$ & $\%$ & Median & \\
\hline Age (year) & & & 11.4 & & & 12 & \\
\hline $\begin{array}{l}\text { Sex } \\
\quad \text { Female } \\
\text { Male }\end{array}$ & $\begin{array}{c}8 \\
10\end{array}$ & & & $\begin{array}{l}29 \\
40\end{array}$ & & & \\
\hline $\begin{array}{l}\text { Clinical manifestations } \\
\text { Fatigue } \\
\text { Fever }\left(>38.2^{\circ} \mathrm{C}\right) \\
\text { Fever duration-day (median) } \\
\text { Cough and/or respiratory distress } \\
\text { Gastrointestinal manifestations } \\
\text { Mucocutaneous involvement } \\
\text { Cardiovascular involvement }\end{array}$ & $\begin{array}{l}10 \\
5 \\
1 \\
3\end{array}$ & $\begin{array}{c}94.4 \\
100 \\
\\
55.5 \\
27.8 \\
5.5 \\
16.6\end{array}$ & 6 & $\begin{array}{c}40 \\
38 \\
30 \\
6 \\
4 \\
-\end{array}$ & $\begin{array}{c}58 \\
55 \\
\\
43.5 \\
8.7 \\
5.8 \\
-\end{array}$ & 3 & $\begin{array}{l}<0.01 \\
<0.01 \\
<0.00\end{array}$ \\
\hline Previously immunosuppressive and/or biologic treatment & 5 & 27.7 & & 21 & 30.4 & & \\
\hline $\begin{array}{l}\text { Laboratory* } \\
\text { Increased CRP }\left(>0.5 \mathrm{mg} / \mathrm{dL}^{*}\right) \\
\text { Lymphopenia }\left(<1,500 / \mathrm{mm}^{3}\right) \\
\text { Thrombocytopenia }\left(<180 \times 10^{3} / \mathrm{mm}^{3}\right) \\
\text { Increased D-Dimer }(>500 \mathrm{ng} / \mathrm{mL}) \\
\text { Ferritin level }(\mathrm{ng} / \mathrm{mL}) \\
\text { Hyperferritinemia }(>684 \mathrm{ng} / \mathrm{mL}) \\
\text { Increased IL-6 }(>5.9 \mathrm{pg} / \mathrm{mL})\end{array}$ & $\begin{array}{c}18 \\
17 \\
6 \\
15 \\
\\
9 \\
14\end{array}$ & $\begin{array}{c}100 \\
94.4 \\
33.3 \\
83.3 \\
50 \\
77.8\end{array}$ & 439 & $\begin{array}{l}37 \\
18 \\
4 \\
8 \\
\\
6 \\
4\end{array}$ & $\begin{array}{c}53.6 \\
26.1 \\
5.8 \\
11.6 \\
\\
8.7 \\
5.8\end{array}$ & 198 & $\begin{array}{l}<0.01 \\
<0.01 \\
<0.01 \\
<0.01 \\
<0.01 \\
<0.01 \\
<0.01\end{array}$ \\
\hline $\begin{array}{l}\text { COVID-19 diagnosis } \\
\text { Via RT-PCR (nasal swab) positivity } \\
\text { Via antibody testing positivity } \\
\text { Via contact history }\end{array}$ & $\begin{array}{c}5 \\
10 \\
3\end{array}$ & & & $\begin{array}{c}69 \\
- \\
-\end{array}$ & & & \\
\hline $\begin{array}{l}\text { Treatments for COVID-19 } \\
\text { Favipiravir } \\
\text { IVIG } \\
\text { Corticosteroids } \\
\text { LMWH } \\
\text { Anti-IL 6 } \\
\text { Anti IL-1 } \\
\text { Plasmapheresis }\end{array}$ & $\begin{array}{c}4 \\
18 \\
14 \\
16 \\
2 \\
4 \\
4\end{array}$ & $\begin{array}{l}22.2 \\
100 \\
77.8 \\
88.9 \\
11.1 \\
22.2 \\
22.2\end{array}$ & & $\begin{array}{l}26 \\
- \\
- \\
- \\
- \\
- \\
-\end{array}$ & $\begin{array}{c}37.7 \\
- \\
- \\
- \\
- \\
- \\
-\end{array}$ & & $\begin{array}{l}\quad- \\
<0.00 \\
<0.00 \\
<0.00 \\
<0.00 \\
<0.00 \\
<0.00\end{array}$ \\
\hline $\begin{array}{l}\text { Outcome } \\
\text { Totally recovery } \\
\text { Admission on intensive care unit } \\
\text { Utilization of mechanical ventilator } \\
\text { Utilization of ECMO } \\
\text { Mortality }\end{array}$ & $\begin{array}{l}17 \\
7 \\
3 \\
1 \\
1\end{array}$ & & & $\begin{array}{l}69 \\
- \\
- \\
- \\
-\end{array}$ & & & $\begin{array}{l}<0.00 \\
<0.00 \\
<0.00 \\
<0.00\end{array}$ \\
\hline
\end{tabular}

MIS-C: Multisystem inflammatory syndrome in children; CRP: C-reactive protein; IL: Interleukin; RT-PCR: Reverse transcriptase-polymerase chain reaction; IVIG: Intravenous immunoglobulin; LMWH: Low-molecular-weight heparin; ECMO: Extracorporeal Membrane Oxygenation. 
was required in a patient who had a prior diagnosis of JIA and had no active complaints, who received IVIG, steroid, plasmapheresis and anakinra treatments due to resistant MIS-C course, and who had decreased myocardial functions during this period.

The COVID-19 pandemic caused exacerbation of systemic disease in 56 children due to medication cessation, postponed drug switch, or recurrent viral infection. Characteristics of these patients were as follows: 26 (46.4\%) females, median age 10.5 years, and median disease duration 2.3 years (range, 1 to 3 ) years. Among them, $15.1 \%$ of all patients were on methylprednisolone, $23.2 \%$ on a bDMARD (methotrexate or azathioprine), 5.3\% on hydroxychloroquine, and $59 \%(\mathrm{n}=33)$ on any biological drug. Most of them were diagnosed with JIA $(n=31)$ and $67.6 \%$ of these patients $(n=21)$ were on biologics. Twelve patients $(22 \%)$ with FMF were on colchicine.

In this study, we evaluated 1,310 patients by telemedicine. If there was any suspicion about the disease, the patient was called to the hospital. A total of 33 patients were seen in our outpatient clinics after telemedicine due to primary diseaserelated or suspected COVID-19 symptoms. Eight patients had a flare of primary disease and their therapy was rearranged. Also, in four patients was suspected of having COVID-19 infection due to respiratory symptoms, but these patients were ruled out. The other 21 patients had no signs of disease exacerbation or COVID-19 after the examination.

\section{DISCUSSION}

In the current study, we shared our experiences the pediatric patients with rheumatic diseases during the COVID-19 outbreak. Previous studies investigated the epidemiological characteristics of children with infection and reported that they had mostly mild-to-moderate upper respiratory tract and gastrointestinal symptoms. ${ }^{7}$ Complicated COVID-19 infection was not reported in pediatric rheumatic patients with or without bDMARDs. ${ }^{8-10}$ In our cohort, $23 \%$ of patients had a fever for five or more days, of respiratory and digestive findings in $46 \%$ and $12.6 \%$, respectively. Also, we observed fatigue in 57 patients. Lymphopenia, decreased albumin and platelets, increased CRP, neutrophils, aspartate aminotransferase, lactate dehydrogenase, and cytokines were reported, particularly in severe patients. ${ }^{19}$ These abnormal blood results were reported in some pediatric patients with a variety of phenotypes. ${ }^{20}$ Based on our experience, these abnormalities can be seen in patients with a severe clinic and a poor outcome.

Although the incidence of COVID-19 in JIA patients is unknown, the frequency of infections tends to increase in these patients. Previous studies reported that there were both antirheumatic drugs and the disease might increase susceptibility prone to infections in patients with JIA. ${ }^{21}$ However, some reports in adult patients with rheumatic diseases showed that the use of anti-tumor necrosis factor (TNF) had a protective effect on the evolution of severe forms. ${ }^{22,23}$ In a report, a case with mild symptoms who used anti-IL-1 blockers during the COVID-19 pandemic was presented. ${ }^{24}$ However, a study done in China found that rheumatic patients infected with COVID-19 might present with more severe symptoms, compared with non-rheumatic patients. ${ }^{6}$ In the current approach guideline, pediatric rheumatic diseases and rheumatic drugs do not pose an increased risk for COVID-19, glucocorticoid therapy can be modified in case of infection, and existing bDMARD treatments should not be discontinued. ${ }^{25}$

In this study, there were 31 JIA patients among COVID-19-suspected patients; these were $1.6 \%$ of all JIA patients evaluated during this period. Sixteen of 31 patients were receiving a biologic with or without methotrexate. Most of the patients using a biologic (76.9\%) had to be hospitalized to receive treatment. In our cohort, while all patients with IL-1 blockers were hospitalized, this rate was $50 \%$ in patients treated with anti-TNF. It was seen from adult studies that patients with and without the rheumatic disease had similar clinical features and hospitalization rates. Besides, the rheumatic patients required intensive care unit admission and mechanical ventilation more often than the others. ${ }^{5,6}$ Ye et al. ${ }^{6}$ reported from China that respiratory failure was more common in rheumatic patients infected with COVID-19. In our study, mild-to-moderate involvement on thoracic CT was observed in 
patients with or without using biological therapy. Our results do not support the protective role of biological drugs, similar to adult studies reported previously. ${ }^{26}$ However, during the pandemic period, we can suggest that there is no risk of side effects that would require the cessation of these medicines.

Overall, MIS-C was reported two to four weeks after infection with SARS-CoV-2. The disorder is rare in all infected pediatric patients over the same period ( 2 vs. 322 in 100,000 persons). ${ }^{12}$ In our patient group, 18 patients were diagnosed with MIS-C, accounting for $0.4 \%$ of all admissions to the rheumatology clinic. This rate is higher than the literature, as our center is a reference clinic and every patient with a suspected MIS-C is evaluated. Five of the patients who received biological treatment and evaluated with suspicion of COVID-19 infection had MIS-C (27.7\%); this rate was found to be $1.3 \%$ among all patients receiving biological treatment. This can be attributed to the fact that the biological drugs used can suppress this hyperinflammatory condition that develops after infection.

Due to the hygiene rules, travel restrictions, and the necessity to stay at home, telemedicine became an effective assessment modality of pediatric rheumatic diseases. ${ }^{10}$ In this period, we had to restrict access to our outpatient clinic, as expected. Nevertheless, we maintained the administration of intravenous drugs. Apart from these patients, we evaluated patients in the outpatient clinic, and we reached 1,310 patients by phone consultation. We checked their clinical status, disease activities, and drug status, and we received information about their exposure to COVID-19 from themselves or their close contacts. Among all evaluated patients, we observed disease exacerbation in 56; most of them were diagnosed with JIA and under biologics during the COVID-19 outbreak. Except for exacerbation of the disease caused by a viral infection, some patients were unable to access the drug and some patients delayed drug switching. Therefore, it seems that telemedicine applications would become increasingly important in the future.

Despite its large sample size, the main limitations of this study is its retrospective design without a control group. Further large-scale, prospective studies are needed to confirm these findings.

In conclusion, pediatric rheumatic patients do not seem to present a higher risk of severe COVID-19, similar to healthy children. Whether these patients receive biological treatment does not affect the severity of the disease; however, it is still not true to conclude that these drugs are protective. Based on our experience, we suggest that care should be taken in terms of a hyperinflammatory syndrome which may be severe in this group of patients and may be confused with primary diseases and, therefore, close monitoring is critical.

\section{Acknowledgment}

We are grateful to all participating children and their families. We would like to also thank our clinical nurse Gülbeyaz Göktuğ, who contributed to the telemedicine interviews.

\section{Declaration of conflicting interests}

The authors declared no conflicts of interest with respect to the authorship and/or publication of this article.

\section{Funding}

The authors received no financial support for the research and/or authorship of this article.

\section{REFERENCES}

1. Guan WJ, Ni ZY, Hu Y, Liang WH, Ou CQ, He JX, et al. China Medical Treatment Expert Group for Covid19. Clinical Characteristics of Coronavirus Disease 2019 in China. N Engl J Med 2020;382:1708-20.

2. COVID-19 (Sars-Cov-2 Enfeksiyonu) bilgilendirme sayfasi; [updated 2021 Jan 9]. Available at: https:// covid19.saglik.gov.tr/TR-68443/covid-19-durumraporu.html

3. Listing J, Gerhold K, Zink A. The risk of infections associated with rheumatoid arthritis, with its comorbidity and treatment. Rheumatology (Oxford) 2013;52:53-61.

4. D'Antiga L. Coronaviruses and immunosuppressed patients: The facts during the third epidemic. Liver Transpl 2020;26:832-4.

5. D'Silva KM, Serling-Boyd N, Wallwork R, Hsu T, Fu $\mathrm{X}$, Gravallese EM, et al. Clinical characteristics and outcomes of patients with coronavirus disease 2019 (COVID-19) and rheumatic disease: a comparative cohort study from a US 'hot spot'. Ann Rheum Dis 2020;79:1156-62. 
6. Ye C, Cai S, Shen G, Guan H, Zhou L, Hu Y, et al. Clinical features of rheumatic patients infected with COVID-19 in Wuhan, China. Ann Rheum Dis 2020;79:1007-13.

7. Dong $\mathrm{Y}$, Mo X, Hu Y, Xin Qi, Jiang F, Jiang Z, et al. Epidemiological characteristics of 2143 pediatric patients with 2019 coronavirus disease in China. Pediatrics. 2020.

8. Filocamo G, Minoia F, Carbogno S, Costi S, Romano M, Cimaz R; Pediatric Rheumatology Group of the Milan Area. Absence of severe complications from SARS-CoV-2 infection in children with rheumatic diseases treated with biologic drugs. J Rheumatol 2020:jrheum.200483.

9. Haslak F, Yildiz M, Adrovic A, Sahin S, Koker O, Aliyeva A, et al. Management of childhood-onset autoinflammatory diseases during the COVID-19 pandemic. Rheumatol Int 2020;40:1423-31.

10. KasapCuceogluM,BatuED, BilginerY,ÖzenS. COVID19 in paediatric rheumatology patients treated with b/ tsDMARDs: a cross-sectional patient survey study. Ann Rheum Dis 2020:annrheumdis-2020-218341. [Online ahead of print]

11. Koker O, Demirkan FG, Kayaalp G, Cakmak F, Tanatar A, Karadag SG, et al. Does immunosuppressive treatment entail an additional risk for children with rheumatic diseases? A survey-based study in the era of COVID-19. Rheumatol Int 2020;40:1613-23.

12. Dufort EM, Koumans EH, Chow EJ, Rosenthal EM, Muse A, Rowlands J, et al. Multisystem inflammatory syndrome in children in New York state. N Engl J Med 2020;383:347-58.

13. Feldstein LR, Rose EB, Horwitz SM, Collins JP, Newhams MM, Son MBF, et al. Multisystem inflammatory syndrome in U.S. children and adolescents. N Engl J Med 2020;383:334-46.

14. Whittaker E, Bamford A, Kenny J, Kaforou M, Jones $\mathrm{CE}$, Shah $\mathrm{P}$, et al. Clinical characteristics of 58 children with a pediatric inflammatory multisystem syndrome temporally associated with SARS-CoV-2. JAMA 2020;324:259-69.

15. Levin M. Childhood multisystem inflammatory syndrome - a new challenge in the pandemic. N Engl J Med 2020;383:393-5.

16. Centers for Disease Control and Prevention. Multisystem inflammatory syndrome in children (MIS-C) associated with coronavirus disease 2019 (COVID-19) [updated
2002 May 14]. Available at: https://emergency.cdc. gov/han/2020/han00432.asp

17. World Health Organization. Multisystem inflammatory syndrome in children and adolescents with COVID19: Scientific brief. 15 May 2020 Statement. Available at: https://www.who.int/publications/i/item/ multisystem-inflammatory-syndrome-in-children-andadolescents-with-covid-19

18. COVID-19 (Sars-Cov-2 Enfeksiyonu) genel bilgiler, epidemioloji ve tan1; [updated 2002 June 29]. Available at: https://covid19bilgi.saglik.gov.tr/tr/ covid-19-rehberi.html

19. Mo P, Xing Y, Xiao Y, Deng L, Zhao Q, Wang H, et al. Clinical characteristics of refractory COVID19 pneumonia in Wuhan, China. Clin Infect Dis 2020:ciaa270.

20. Lu X, Zhang L, Du H, Zhang J, Li YY, Qu J, et al. SARS-CoV-2 infection in children. $\mathrm{N}$ Engl $\mathrm{J}$ Med 2020;382:1663-5.

21. Licciardi F, Giani T, Baldini L, Favalli EG, Caporali R, CimazR. COVID-19and what pediatric rheumatologists should know: a review from a highly affected country. Pediatr Rheumatol Online J 2020;18:35.

22. Brito CA, Paiva JG, Pimentel FN, Guimarães RS, Moreira MR. COVID-19 in patients with rheumatological diseases treated with anti-TNF. Ann Rheum Dis 2020. [Online ahead of print]

23. Gianfrancesco M, Hyrich KL, Al-Adely S, Carmona L, Danila MI, Gossec L, et al. Characteristics associated with hospitalisation for COVID-19 in people with rheumatic disease: data from the COVID-19 Global Rheumatology Alliance physician-reported registry. Ann Rheum Dis 2020;79:859-66.

24. Moutsopoulos HM. Anti-inflammatory therapy may ameliorate the clinical picture of COVID-19. Ann Rheum Dis 2020;79:1253-4.

25. Wahezi DM, Lo MS, Rubinstein TB, Ringold S, Ardoin SP, Downes KJ, et al. American college of rheumatology guidance for the management of pediatric rheumatic disease during the COVID19 pandemic: Version 1. Arthritis Rheumatol 2020;72:1809-19.

26. Conticini E, Bargagli E, Bardelli M, Rana GD, Baldi C, Cameli P, et al. COVID-19 pneumonia in a large cohort of patients treated with biological and targeted synthetic antirheumatic drugs. nn Rheum Dis 2021;80:e14. 